\title{
EDITORIAL
}

\section{The murky world of disclosures}

if any level of doubt exists regarding whether or not an interaction is deemed to be a competing interest, it needs to be declared.
Many of our readers will be aware of the recent resignation of Jose Baselga from his position as Chief Medical Officer at Memorial Sloan Kettering Cancer Center, following a failure to declare numerous competing interests in clinical trial reports published in high-profile journals ${ }^{1}$. Jose Baselga most likely did not deliberately intend to mislead the oncology community, or the general public, regarding his competing interests; nor is he likely to be the only oncologist to have failed to declare such interests. Nonetheless, the consequences of these omissions highlight the need to re-open the debate surrounding the influence of competing interests in cancer research and treatment.

The source of this issue lies in how we develop anticancer drugs. Clinical trials and the prospective evidence they provide are the cornerstone of evidence-based medicine. Such trials are also expensive to conduct and carry no guarantee of success. With these obvious caveats in mind, such trials are mostly funded by the manufacturer of the drug. Thus, even when declared as having no role in trial design, for-profit companies have a profound influence on the treatment of patients with cancer: they are the suppliers of virtually all anticancer drugs. Furthermore, oncologists in many institutions will be encouraged to work closely with such companies to develop novel, and hopefully better, interventions.

These interactions are, to some extent, unavoidable. How else would the pharmaceutical industry be able to develop the most effective medicines without the valued input of experienced oncologists? Accordingly, oncologists often receive payments for their contributions - such as serving as consultants or advisory board members to the trial sponsors, or honoraria for other services - and will also go on to prescribe such medications and other interventions in their clinical practice. Hence, the competing interests need to be clearly stated.

In the current political and economic climate, competing interests will always exist to some extent: but, do these really influence clinical practice? They quite possibly do. In an analysis of the effect of 'financial ties', (which included, among others, consultancy payments, honoraria, and stock options) in 195 randomized controlled trials designed to assess efficacy (22 in oncology) ${ }^{2}$, a statistically significant association was reported between the presence of a financial tie between the principal investigator of the trial and the trial sponsor and a positive outcome. In fact, trials for which this link existed were about three times as likely to have positive results ${ }^{2}$. However, this was a cross-sectional analysis and, as concerning as the findings may seem, these should not, alone, be taken as evidence of causation. As another example, while we do not believe any oncologist would deliberately prescribe an intervention based primarily upon the generosity of the manufacturer, why did pharmaceutical companies spend A $\$ 286,117,928$ (>US\$200,000,000 at current exchange rates) in the period October 2011 to September 2015 on industry-sponsored events for health professionals in Australia alone ${ }^{3}$ The true extent to which these competing interests, however small or substantial, influence medical decisions seems impossible to determine, although the recent resignation of Jose Baselga highlights that society does consider this an important issue.

Just as the effects of competing interests can be difficult to define, for editors, such interests, if undeclared, can also be difficult to detect. For this reason, Nature Reviews Clinical Oncology and many other journals will most likely continue to rely upon self-declared competing interests. We, like many other journals, do not have the resources to routinely investigate the competing interest statement of each author; although, perhaps now is the time to consider industry-wide solutions that might enable all journals to address this challenge.

For these reasons, we, the editors, wish to clarify that Nature Reviews Clinical Oncology, similar to other Nature Research titles, requires all potential competing financial interests broadly relevant to an article to be declared. Non-financial competing interests, such as unpaid roles on advisory boards or acting as an expert witness, should also be considered possible conflicts of interest, and all possible competing interests should be disclosed before the start of the peer review process ${ }^{4}$.

For those publishing in journals that currently do not have defined positions, a simple piece of advice is that if any level of doubt exists regarding whether or not an interaction is deemed to be a competing interest, it needs to be declared. A failure to take this issue seriously could have severe consequences: ultimately, every reader must be allowed to decide for themselves what is, and what is not, an acceptable conflict of interest.

\footnotetext{
1. Thomas, K. \& Ornstein, C. Top Sloan Kettering Cancer doctor resigns after failing to disclose industry ties. New York Times https://www.nytimes.com/2018/09/13/health/jose-baselga-cancermemorial-sloan-kettering.html (2018).

2. Ahn, R. et al. Financial ties of principal investigators and randomized controlled trial outcomes: cross sectional study. BMJ 356, i6770 (2017)

3. Fabbri, A. et al. A cross-sectional analysis of pharmaceutical industry-funded events for health professionals in Australia. BMJ Open 7, e016701 (2017).

4. Nature. Nature Research journals' competing interests policy. Nature https:/www.nature.com/authors/policies/competing.html (2018).
} 